\title{
Experimental and numerical investigation of fractal-tree-like heat exchanger manufactured by 3D printing
}

\author{
Gang Wang ${ }^{a}$, Yu Gu ${ }^{a}$, Luhaibo Zhao ${ }^{a}$, Jin Xuan ${ }^{b}$, Gaofeng Zeng ${ }^{a, c}$, \\ Zhiyong Tang ${ }^{\mathrm{a}, \mathrm{c}^{*}}$, Yuhan Sun ${ }^{\mathrm{a}, \mathrm{c}^{*}}$
}

a. CAS Key Laboratory of Low-Carbon Conversion Science and Engineering, Shanghai Advanced Research Institute (SARI), Chinese Academy of Sciences (CAS), Shanghai 201210, China

b. Institute of Mechanical, Process and Energy Engineering, School of Engineering and Physical Sciences, Heriot-Watt University, Edinburgh, EH14 4AS, UK

c. School of Physical Science and Technology, ShanghaiTech University, Shanghai 201210, China

* Corresponding authors

E-mail address: tangzy@,sari.ac.cn (Z. Tang); sunyh@,sari.ac.cn (Y. Sun) 
Abstract: The manufacturing difficulties of complex fractal-tree-like heat exchangers have limited their industrial applications, although many evidences have shown that they have significant advantages in heat transfer. Nevertheless, the emerging 3D printing technology has brought great opportunity for the development of complex structured device. In the present study, three-dimensional (3D) fractal-tree-like heat exchangers were designed and manufactured using 3D printing technology. Their performance was evaluated from both thermal and hydrodynamic perspectives, the flow characteristics were investigated in detail. The results show that a fractal-tree-like heat exchanger can improve hydrodynamic performance, reduce pressure drops and has great heat transfer ability. In general, the fractal-tree-like heat exchanger has a comprehensive advantage over the traditional spiral-tube exchangers as it has a higher value of coefficient of performance (COP). Furthermore, the 3D printing provides a visual, efficient, and precise approach in the present research.

Key words: Fractal-tree-like, heat exchanger, heat transfer, 3D printing, Computational Fluid Dynamics (CFD) simulation

\section{Introduction}

In recent years, the demand for $\mathrm{CO}_{2}$ reduction has been increasing in various industries. An International Energy Agency (IEA) report states that as a major contributor to climate change, global $\mathrm{CO}_{2}$ emissions reached $32.2 \mathrm{Gt}$ in 2013 , and will reach $38.03 \mathrm{Gt}$ in $2040^{[1,2]}$. At present, increasing energy efficiency and $\mathrm{CO}_{2}$ Capture, Utilization, and Storage (CCUS) technology ${ }^{[3]}$ are two important ways to reduce $\mathrm{CO}_{2}$ emissions. As an important device of chemical industry, heat exchanger is widely used 
in the synthetic ammonia industry, sulfuric acid industry, petroleum, etc. It has a significant effect on the efficient use of energy in $\mathrm{CO} 2$ capture and utilization. For instance, heat exchanger can help $\mathrm{CO}_{2}$ hydrogenation (a highly exothermic reaction) to reach equilibrium by removing reaction heat efficiently, and hence improves the process efficiency ${ }^{[4]}$. In addition, heat recovery through heat exchanger during liquid-absorbent-based carbon capture helps to achieve considerable energy savings. Therefore, much attention has been devoted to improving the efficiency of the heat exchanger. Novel designs especially fractal-tree-like heat exchangers appear to have great advantages over traditional tube-shell or tube-spin heat exchangers.

The "fractal-tree-like" concept was first proposed by Adrain Bejan and M. R. Errera ${ }^{[5]}$, which has been applied to various chemical equipments such as stirrers ${ }^{[6]}$, distributors ${ }^{[7]}$, and reactors ${ }^{[8]}$. The concept has also been adopted in the design of a heat exchanger [9]. Subsequently, Chen and Cheng ${ }^{[10-12]}$ designed a sandwich-structured fractal-tree-like heat sink and compared it with traditional serpentine nets. The researchers assumed that the flow was laminar, and neglected the pressure drop during bifurcation. Numerically and experimentally, they found that their new fractal branching channel net had a stronger heat transfer capability and required lower pumping power.

Yu et al. ${ }^{[13]}$ investigated the hydraulic and thermal characteristics of fractal-tree-like rectangular microchannels with different aspect ratios (ARs) for Reynolds numbers ranging from 150 to 1200 . Their observations revealed that fractal-tree-like microchannels could achieve higher heat transfer efficiency compared 
with straight heat exchanger, but at the cost of higher pump power. In addition, the ARs had a significant influence on the performance of fractal-tree-like microchannels in terms of pressure drop and heat transfer.

Luo et al. ${ }^{[14]}$ designed a multifunctional hierarchical multichannel mini heat-exchanger reactor with an arborescent distributor and collector. Two different fluids were divided into 16 channels through the distributor, and then mixed at the inlet of each channel, which led to a uniform mixture. With regard to the heat exchanger ${ }^{[8]}$, a high value of the heat transfer coefficient was obtained, suggesting an end effect and non-established flow.

M. O. Coopens studied the mechanisms used by nature-inspired reactors to solve fundamental problems in chemistry engineering, such as scalability, efficiency and robustness ${ }^{[15]}$. These mechanisms include: (a) hierarchical networks are often used in biology to bridge scales and facilitate transport, leading to efficient and scalable solutions; (b) careful balancing of forces at multiple scales can achieve superior performance; (c) nature employs dynamics to form complex organizations from simple components.

Most of the above studies were mainly conducted at the microscale in two dimensions, with the smallest length between $10 \mu \mathrm{m}$ and $200 \mu \mathrm{m}^{[16-17]}$. Investigations of 3D fractal-tree-like structure heat exchange were carried out mainly via modeling or CFD simulation owing to restrictions in manufacturing technology ${ }^{[18]}$. The hydraulic and thermal characteristics of a 3D fractal-tree-like heat exchanger in a relative larger scale have not yet been studied experimentally. In actual practice, the 
fluid mechanisms varies with different scales of the system, the performance of the heat exchange in larger scale cannot be simply extrapolated from the data obtained in the microscale system. Therefore it is essential to investigate the flow performance in a wider range of system configurations in order to gain a comprehensive understanding of the heat exchange in complex networks.

Recently, 3D printing technology has developed rapidly and been applied in many fields ${ }^{[19]}$. It has great potential in the manufacture of complex fractal structures with high precision and less limitation of size. Furthermore, 3D printing is a simple and efficient method to investigate the structure-activity relationships in a structure, an important topic in the researches of heat exchangers ${ }^{[20]}$, and to produce any feasible product without having to first create a costly production-grade mode or tool ${ }^{[21]}$. In effect, new ideas in the design and manufacture of heat exchangers, including 3D printing, present a very exciting frontier. Three types of 3D printers are commonly used: stereo lithography (SLA), fused deposition modeling (FDM), and selective laser sintering (SLS) ${ }^{[22]}$. Among these, the SLA printer has proven to be efficient, accurate, and precise. It can print transparent channels with smooth surfaces, which enables optical access to flow phenomena. Therefore, we chose an SLA 3D printer in the present research.

In the current work, we designed and printed three types of 3D fractal-tree-like heat exchangers: Y-type, H-type, and the traditional spiral-tube type. The same surface area for heat transfer was applied in each design and the fluid used in the experiment was deionized water. We analyzed the performance of fluid flow in the 
fractal-tree-like channels carefully, and investigated the energy dissipation and heat transfer performance of each heat exchanger both experimentally and numerically. Furthermore, we also discussed the integration of 3D printing technology for smart manufacturing of special chemical equipment.

\section{Physical and numerical models}

\subsection{Description of experimental facility}

\subsubsection{Fractal-tree-like heat exchanger fabricated using a 3D printer}

Three types of heat exchangers were fabricated by 3D printer with photosensitive resin model VisiJet ${ }^{\circledR}$ SL Clear by SLA technology, as illustrated in Fig. 1, including two fractal-tree-like heat exchangers and one traditional spiral-tube heat exchanger. These exchangers were made of photosensitive resin with a wall thickness of $1.5 \mathrm{~mm}$, and have constant surface area. The SLA 3D printer used in this study was the model ProJet ${ }^{\circledR} 7000 \mathrm{HD}$ made by the 3D Systems company, whose accuracy can be controlled within $0.025-0.05 \mathrm{~mm}$.

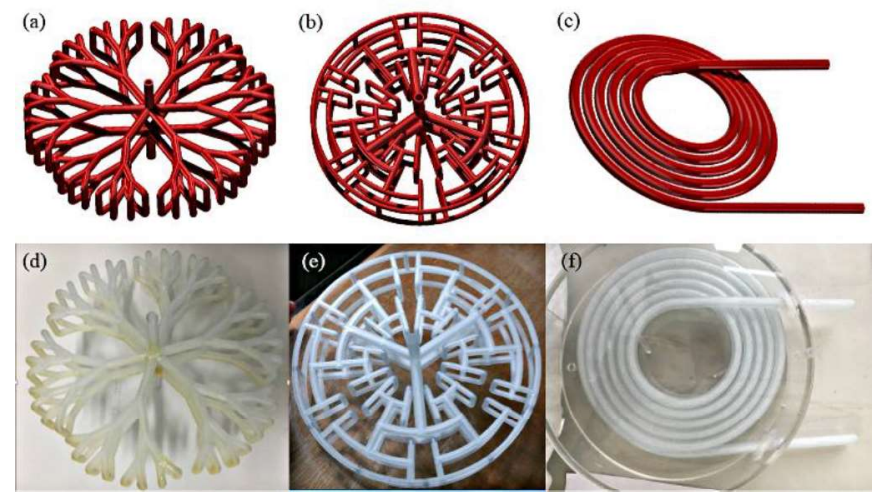

Fig.1 CAD diagram and photos of three heat exchangers: (a), (d) Y-type; (b), (e) H-type; and (c), (f) traditional spiral-tube 
According to Murray's law ${ }^{[23]}$, the geometry of fractal structure would be in the optimal state in the aspect of the pressure drop, as long as it satisfies the relationship of Eq. (1)

$$
d_{i-1}^{3}=d_{i 1}^{3}+d_{i 2}^{3}
$$

Where $d_{i-1}$ is the diameter of the parent channel in level $i-1 ; d_{i 1}$ and $d_{i 2}$ are the diameters of the daughter channels in level $i$, respectively. An equation can show the diameter relationship between two adjacent levels (parent and daughter channels), which is $\frac{d_{i-1}}{d_{i}}=N^{1 / \Delta}$. In the model, each channel is divided into two branches at the next level, that is, $\mathrm{N}=2 . \Delta$ is the so-called fractal dimension of the hydraulic diameter $^{[12]}$.

However, this symmetric structure may lead to the intersection of some branches in the engineering design and make the network not completely compact, since the ratios of the fractal diameters obey Murray's law. Therefore, we modified the fractal heat exchanger, drawing inspiration from trees. The "tree", also known as "nonuniform fractals" is the sum of parts of varying fractal and topological dimensions. A tree designer may either allow some branched to grow or write a more complicate program to instruct these branches never to grow ${ }^{[24]}$. Similarly, we carefully modified the lengths, diameters, and angles of the bifurcation as shows in Fig. 2. The inlet channel is a single tube with diameter $d_{0}$. The geometry for the secondary, tertiary, and lower levels of branching is symmetric, i.e., $d_{i 1}=d_{i 2}$. The radii ratio of adjacent levels was set as 1.26 (for even level) and 1.414 (for odd level) for H-type heat exchanger. A more specific ratio of 1.17 was chosen for the diameter ratio 
in the Y-type exchanger to avoid channel overlapping and to improve the compactness of the networks. The radii ratio, length ratio and the angle between two daughter channels can affect the flow characteristic and heat transfer in certain extent, but the investigation of these parameters is out of the present research scope and can be considered as future study. The geometries of the fractal-tree-like exchanger used in simulation and experiment are constant, and the structural parameters of the Y-type and H-type heat exchangers are summarized in Table 1, in which it can be seen that the maximum and minimum channel diameters of the Y-type and H-type heat exchangers are $4.8 \mathrm{~mm} / 3 \mathrm{~mm}$ and $6.33 \mathrm{~mm} / 2 \mathrm{~mm}$, respectively. The level-4 length of the H-type exchanger was not listed in the table to avoid adding excessive detail in the definition. In addition, the radii of the Y-type, H-type, and spiral-type heat exchangers are $104.8 \mathrm{~mm}, 105.3 \mathrm{~mm}$, and $107.6 \mathrm{~mm}$, respectively. The inner specific surfaces of the $\mathrm{Y}, \mathrm{H}$, and spiral-type exchangers are $1.15 \mathrm{~mm}^{2} / \mathrm{mm}^{3}, 1.23 \mathrm{~mm}^{2} / \mathrm{mm}^{3}$, and $0.57 \mathrm{~mm}^{2} / \mathrm{mm}^{3}$, respectively.
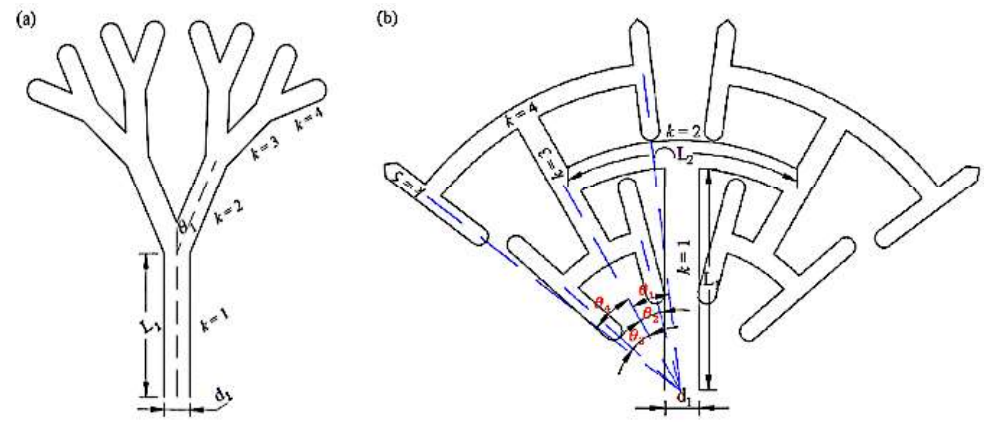

Fig.2 Nomenclature of tree-like flow passages of (a) Y-type and (b) H-type heat exchangers 
Table 1. Structural parameters of two fractal-tree-like heat exchangers

\begin{tabular}{ccccccc}
\hline & \multicolumn{2}{c}{$L(\mathrm{~mm})$} & \multicolumn{2}{c}{$D(\mathrm{~mm})$} & $\left(^{\circ}\right)$ \\
\cline { 2 - 6 } Level $k$ & Y-type & H-type & Y-type & H-type & Y-type & H-type \\
\hline 1 & 40 & 65 & 4.8 & 6.33 & 24.5 & 20 \\
2 & 28.6 & 45 & 4.11 & 4.48 & 22 & 15 \\
3 & 19.6 & 30 & 3.51 & 3.56 & 22.5 & 19 \\
4 & 13.8 & --- & 3 & 2.52 & --- & 23 \\
5 & --- & 25 & --- & 2 & --- & -- \\
\hline
\end{tabular}

The Reynolds number of the tube side is defined as

$$
R e=\frac{\mathrm{du}}{\mathrm{v}}
$$

Where, $\mathrm{d}$ is the diameter of the channel, $\mathrm{u}$ is the flow velocity and $v$ is the kinematic viscosity of the flow medium. Since the diameters along the fractal-tree-like exchangers vary, the Reynolds number subsequently varies. The criterion for the Reynolds number should be set to be constant, so that it can ensure the consistency of external conditions. The diameters of the branch channels of each heat exchanger are different, as given in Table 1. Nevertheless, the diameter of the inlet channel of the three types of heat exchangers is constant at $7 \mathrm{~mm}$. The Reynolds number of the entrance is used to compare the differences in performance between different types of heat exchangers in the experiment and simulation, and is defined as the device Reynolds number $\operatorname{Re}_{d}{ }^{[25-26]}$

The Reynolds number for each channel is defined as the local Reynolds number $R e_{l}$, to distinguish it from $R e_{d}$. We can examine $R e_{l}$ of two adjacent-level branch channels of fractal-tree-like heat exchangers $R e_{i} / R e_{i+l}$, according to Eq. (2)

$$
\frac{R e_{i}}{R e_{i+1}}=\frac{d_{i} u_{i}}{d_{i+1} u_{i+1}}=\frac{d_{i} \frac{V_{i}}{S_{i}}}{d_{i+1} \frac{V_{i+1}}{S_{i+1}}}
$$


Where $R e_{i}, V_{i}$ and $\mathrm{S}_{\mathrm{i}}$ is the Reynolds number, volumetric flow rate and the cross-sectiWonal area of branch $i$, and the $S_{i}$ is equal to $\pi\left(\frac{d_{i}}{2}\right)^{2}$. Thus, Eq. (3) can be written as follows.

$$
\frac{R e_{i}}{R e_{i+1}}=\frac{V_{i} d_{i+1}}{V_{i+1} d_{i}}
$$

According to the principle of mass conservation, $V_{i} / V_{i+1}=2$, and the value of $d_{i+1} / d_{i}$ is always higher than $1 / 2$ according to Table 1 . Thus, the value of Eq. (4) is always higher than 1 , which means that as the branch levels increase, $R e_{l}$ in the fractal-tree-like channels decreases.

The flow rate of the tube side ranges from $10 \mathrm{~L} / \mathrm{h}$ to $100 \mathrm{~L} / \mathrm{h}$. According to the discussion above, the calculated $R e_{l}$ of the vast majority of channels is less than 2000, which indicates a flow in the laminar zone. In cases at a high flow rate, only a few channels have a $R e_{l}$ higher than 2000 (see Table S1) and exist in the transition zone of the laminar flow and turbulent flow. Since this situation is rare, the flow can be considered to be in the laminar zone over the scales.

\subsubsection{Test section description}

The experimental rig contains two closed flow loops (shell side and tube side) and a test section. The thermal tolerance of the device material is approximately $60^{\circ} \mathrm{C}$; hence, a temperature range of $10^{\circ} \mathrm{C}$ to $40^{\circ} \mathrm{C}$ was selected. A schematic diagram of the experimental setup is shown in Fig. 3 (a). Hot water passes through the tube side, whereas cold water passes through the shell side. Fig. 3 (b) shows the schematic of the test section. The solid arrow points to the hot-water circulation path (tube side), while the hollow arrow points to the import and export of cold water (shell side). The 
red line represents a hot-water flow loop consisting of a precise low-temperature thermostat, centrifugal magnet pump, flow meter, thermocouple, differential pressure transducer, and the tube side of the heat exchanger. To avoid the effect of additional pressure drop, the pressure probe was set near the inlet and outlet as soon as possible. The blue line represents the cold water path, which contains a pump. The shell of the heat exchanger was made of polymethyl methacrylate (PMMA). The shell side and the tube side were connected via ultraviolet (UV) glue to prevent water leaking from the interface.

(a)
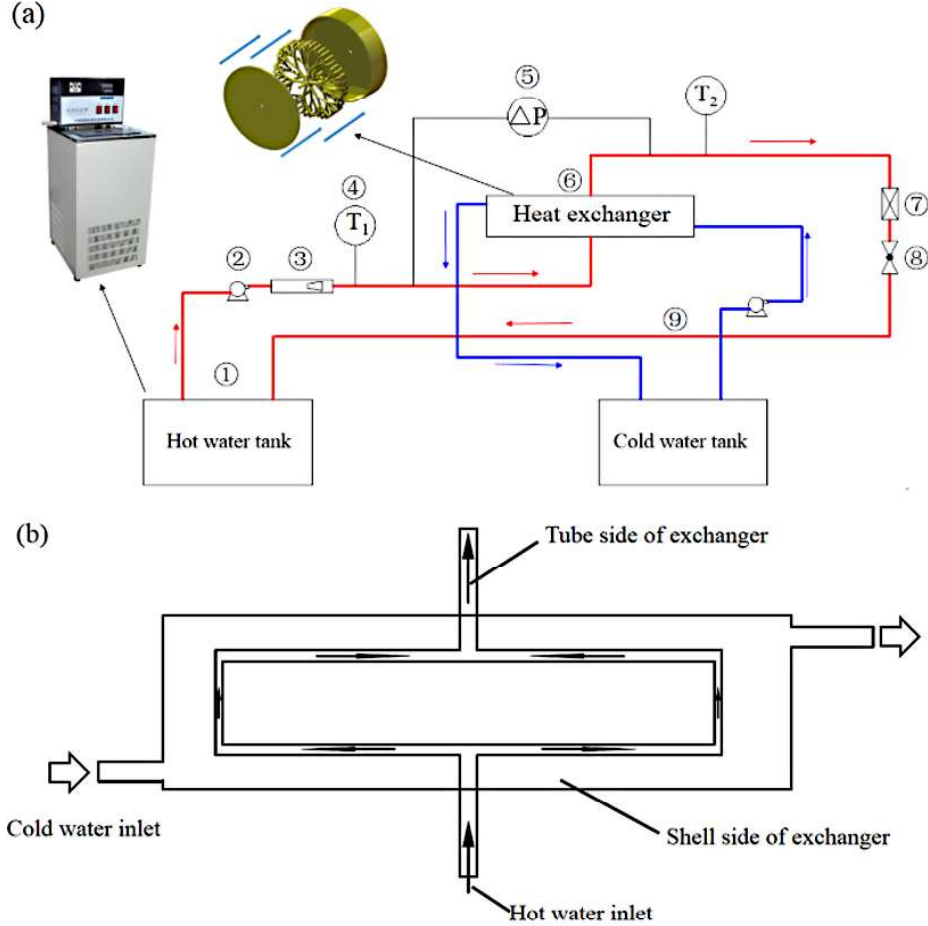

Fig.3 Schematic diagram of (a) experimental setup: (1) low-temperature thermostat, (2) centrifugal magnet pump, (3) flowmeter, (4) K-type thermocouple, (5) differential pressure transducer, (6) heat exchanger, (7) regulating valve, (8) ball valve, (9) rubber hose, and (b) test section

Three main types of measuring or controlling devices were used in the experiment. The temperature of the working medium was measured with a K-type thermocouple (Omega), which was calibrated by the "oil bath calibration method." 
The experimental details are available in the references ${ }^{[27-28]}$. The flow rate of the cold water was kept constant at the maximum flow rate of the thermostat, at approximately $6 \mathrm{~L} / \mathrm{min}$. Because of the high flow rate and the large volume of the shell side, the temperature of the shell side was assumed to stay constant. On the other hand, the experimental temperature difference between the inlet and the outlet of the shell side ranged from $0.2{ }^{\circ} \mathrm{C}$ to $0.4{ }^{\circ} \mathrm{C}$. The temperature of the shell side was considered to be constant at $283 \mathrm{~K}$.

\subsection{Numerical model}

Numerical simulations were performed to investigate the performance of the heat exchanger. Computation Fluid Dynamic (CFD) simulation tool FLUENT 12.0 was employed to calculate the pressure drop, temperature difference, and velocity profile of the heat exchangers. The liquid used in this study was deionized (DI) water, whose physical properties are listed in Table $2^{[29]}$. The Grashof number ${ }^{[30]}$ was evaluated for all scales, and the effect of natural convection was confirmed to be negligible. From an analysis of $R e_{l}$, since the flow in the fractal channels is in the laminar zone over the scales, the laminar model was selected. In effect, both the laminar and RANS (both $k-\varepsilon$ and $k-\omega$ ) models were employed to account for the single-phase liquid flow, and we found that the results of the laminar model were the closest to the experimental results. The gravitational acceleration was set as $-9.8 \mathrm{~m} / \mathrm{s}^{2}$ in the Z-axis direction, because the hot water flows from the bottom up. The pressure and velocity were coupled with the SIMPLE algorithm. The convergence criteria are that the velocity and continuity residuals are less than $1 \times 10^{-6}$ for the continuity equations, 
and $1 \times 10^{-8}$ for the energy equation. The boundary conditions for the tube side are as follows:

(1) Inlet: the type of inlet was a velocity inlet, the inlet temperature was set to be constant at $313 \mathrm{~K}$, and the velocity at the inlet varied from $0.072 \mathrm{~m} / \mathrm{s}$ to $0.722 \mathrm{~m} / \mathrm{s}$, corresponding to a volumetric flow rate of $10 \mathrm{~L} / \mathrm{h}$ to $100 \mathrm{~L} / \mathrm{h}$.

(2) Wall: the temperature of the wall was set constant at $283 \mathrm{~K}$, the material was defined as a photosensitive resin with a thermal diffusion coefficient of 0.127 $\mathrm{mm}^{2} / \mathrm{s}$ and a density of $1.3 \mathrm{~g} / \mathrm{cm}^{3}$, the specific thermal capacity $C_{p}$ was $1817 \mathrm{~J} \mathrm{~kg}^{-1}$ $\mathrm{K}^{-1}$, and the thickness of the wall was set at a constant value of $0.0015 \mathrm{~m}$.

(3) Outlet: since the fluid flow out of the channel naturally, the model was incompressible and the density of fluid was kept constant, and the outflow was selected as the type of outlet.

Table 2. Physical properties of DI water

\begin{tabular}{lccc}
\hline$\rho\left(\mathrm{kg} \mathrm{m}^{-3}\right)$ & $\lambda\left(\mathrm{W} \mathrm{m}^{-1} \mathrm{~K}^{-1}\right)$ & $C_{p}\left(\mathrm{~J} \mathrm{~kg}^{-1} \mathrm{~K}^{-1}\right)$ & $\mu(\mathrm{Pa} \mathrm{S})$ \\
\hline & $-0.829+7.9 \times 10^{-3} \mathrm{~T}-$ & $5348-7.42 \mathrm{~T}$ & $0.0194-1.065 \times 10^{-4} \mathrm{~T}$ \\
994.2 & $1.04 \times 10^{-5} \mathrm{~T}^{2}$ & $+1.17 \times 10^{-2} \mathrm{~T}^{2}$ & $+1.489 \times 10^{-7} \times \mathrm{T}^{2}$ \\
\hline
\end{tabular}

The finite volume method (FVM) was used as a numerical algorithm to solve the continuity and momentum equations so that we could obtain the numerical results of the pressure drop and velocity distribution. The continuity equation is given as

$$
\frac{\partial(\rho u)}{\partial x}+\frac{\partial(\rho v)}{\partial y}+\frac{\partial(\rho w)}{\partial z}=0
$$

The momentum equations are given as

$$
u \frac{\partial u}{\partial x}+v \frac{\partial u}{\partial y}+w \frac{\partial u}{\partial z}=-\frac{1}{\rho} \frac{\partial P}{\partial x}+\frac{\mu}{\rho}\left(\frac{\partial^{2} u}{\partial x^{2}}+\frac{\partial^{2} u}{\partial y^{2}}+\frac{\partial^{2} u}{\partial z^{2}}\right)
$$




$$
\begin{gathered}
u \frac{\partial v}{\partial x}+v \frac{\partial v}{\partial y}+w \frac{\partial v}{\partial z}=-\frac{1}{\rho} \frac{\partial P}{\partial x}+\frac{\mu}{\rho}\left(\frac{\partial^{2} v}{\partial x^{2}}+\frac{\partial^{2} v}{\partial y^{2}}+\frac{\partial^{2} v}{\partial z^{2}}\right) \\
u \frac{\partial w}{\partial x}+v \frac{\partial w}{\partial y}+w \frac{\partial w}{\partial z}=-\frac{1}{\rho} \frac{\partial P}{\partial x}+\frac{\mu}{\rho}\left(\frac{\partial^{2} w}{\partial x^{2}}+\frac{\partial^{2} w}{\partial y^{2}}+\frac{\partial^{2} w}{\partial z^{2}}\right)+F_{z}
\end{gathered}
$$

The energy equation is given as

$$
u \frac{\partial T}{\partial x}+v \frac{\partial T}{\partial y}+w \frac{\partial T}{\partial z}=\frac{\lambda}{\rho C_{p}}\left(\frac{\partial^{2} T}{\partial x^{2}}+\frac{\partial^{2} T}{\partial y^{2}}+\frac{\partial^{2} T}{\partial z^{2}}\right)+Q_{i}
$$

All of the above calculations were under the assumptions that

(1) The flow is laminar and the flow field is steady;

(2) The fluid is Newtonian and incompressible;

(3) The thermal radiation effect is ignored.

The first order upwind difference scheme was applied firstly to achieve a stable state and to avoid non-convergence. The second order upwind difference scheme was then employed to improve the precision of the calculations further. An unstructured grid was chosen for the present study owing to the complex structure, and a prism grid of the boundary layer was highlighted to capture the boundary phenomenon precisely. A grid sensitivity study was performed, 2.07 million cells for the fractal structure and 0.98 million cells for the traditional spiral structure were chosen after the grid independence test.

\section{Results and discussion}

\subsection{Experimental uncertainties}

The experimental uncertainties of this work were evaluated by using the methods presented by Moffat ${ }^{[31]}$. The uncertainties of a result $\left(W_{R}^{+}\right)$can be defined as follows

$$
W_{R^{+}}=\sqrt{\left(\frac{\partial R^{+}}{\partial x_{1}} W_{x_{1}}\right)^{2}+\left(\frac{\partial R^{+}}{\partial x_{2}} W_{x_{2}}\right)^{2}+\cdots+\left(\frac{\partial R^{+}}{\partial x_{n-1}} W_{x_{n-1}}\right)^{2}+\left(\frac{\partial R^{+}}{\partial x_{n}} W_{x_{n}}\right)^{2}}
$$

Where $R^{+}=f\left(x_{1}, x_{2}, \ldots, x_{n}\right)$ and $x_{n}$ is the variable that affects the results of $R^{+}$. 
Objectively, the channel diameter has a significant influence on the experimental uncertainties. However, the precision of the channel diameter is decided only by the 3D printer. The uncertainty in the diameter and the roughness of the channel were detected with a scanning electronic microscope (SEM), whose results are shown in Fig. 4. From the 100x amplification of the area marked with a red square, we can see that the maximum deviation is approximately $30 \mu \mathrm{m}$. Therefore, the uncertainty in the diameter ranges from $0.6 \%$ to $1.5 \%$. Typically, in the region of laminar flow, the wall roughness is considered negligible to the friction factors, however, many investigations indicated that this may not be entirely accurate especially dealing with the fluid flow and heat transfer ${ }^{[32-33]}$. Decreasing the diameter of the channel leads to a large area to volume ratio in microfluid device, causing a more significant surface effect than that in macroscopic device. The surface effect can enhance both heat transfer and laminar flow, which has been verified both experimentally and numerically ${ }^{[34-35]}$.

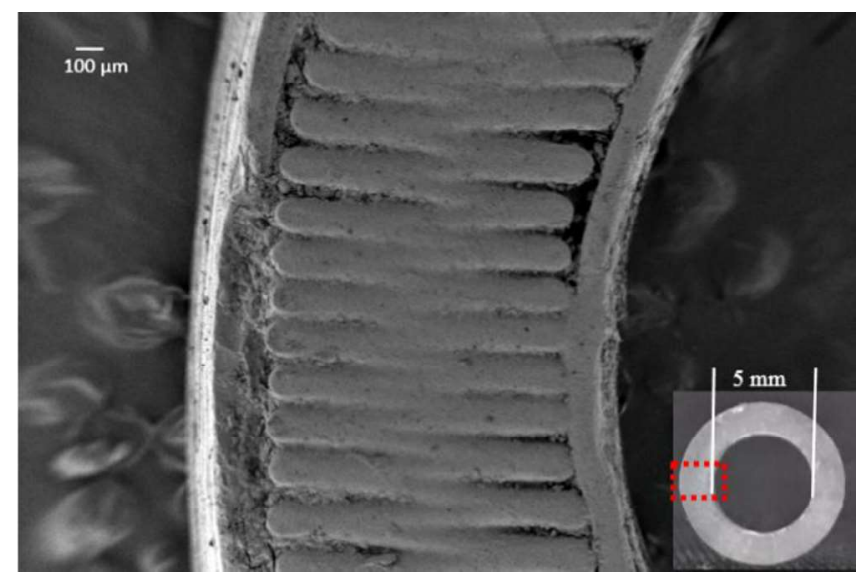

Fig.4 SEM image of the 3D printing tube in the cross-section

The experimental uncertainty of the K-type thermocouple was $\pm 0.23^{\circ} \mathrm{C}$. The 
pressure drop was measured using a differential pressure transducer (PX2300 Series, Omega) with an accuracy of $\pm 0.25 \%$. The inflow passed through an FLR1011 Series flowmeter (Omega) with an accuracy of $\pm 1 \%$. The maximum uncertainties in the Reynolds number, friction factor, pressure drop, and heat flux were estimated to be less than $1.83 \%, 5.78 \%, 5.21 \%$, and $7.69 \%$, respectively.

\subsection{Performance of the flow distribution}

Theoretically, the fractal-tree-like channels encourage a uniform flow distribution owing to their symmetrical structure, in which the fluid is separated into two uniform channels at a bifurcation point and continues to develop until it reaches the secondary bifurcation point.

The numerical simulation results of transverse flow profiles in two fractal-tree-like heat exchangers are shown in Fig.5. The status of fluid flow in the fractal-tree-like channels is rather complex because the length in the channels is restricted, which is not enough for fluids to redevelop steadily in terms of fluid dynamics. In the diffluent condition of Fig. 5(a) (b), asymmetric flow is found in the level 3 and level 4 channel. Under the influence of inertia, in the channel of 2 and lower levels, the fluids with highest velocity are generally located in the vicinity of the channel wall. And then the fluids impact against the wall located in front of flow direction, leading to an immediate segregation of velocity vector and uneven splitting of the flow. Consequently, the flow rate at the "daughter" branch channel increases in the natural direction of the "parent" channel. With the development of flow, the flow rate of channels at the same level is not equal ${ }^{[36]}$. 


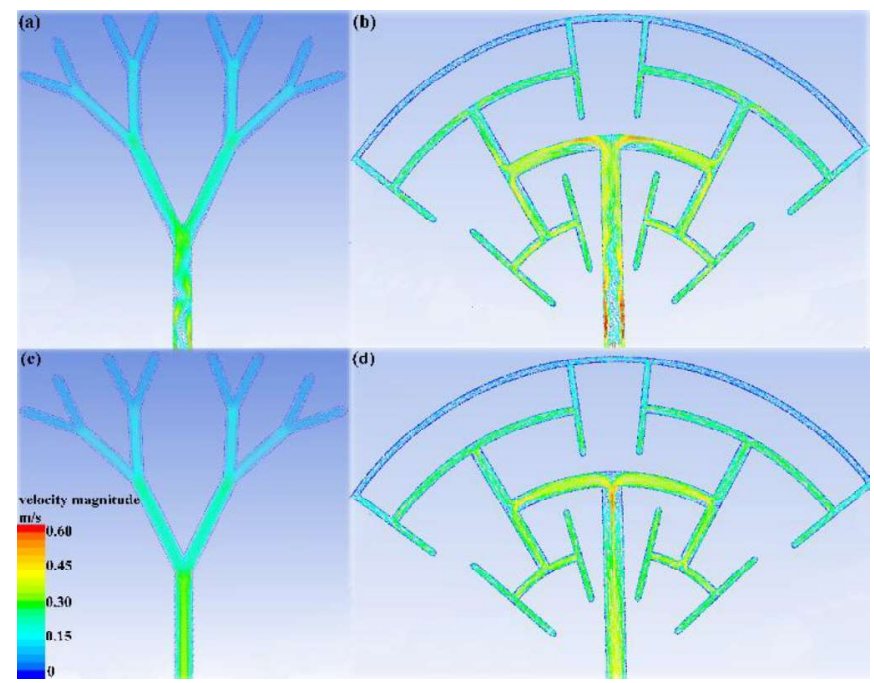

Fig.5 The velocity profiles in the transverse surfaces of the Y and H-type heat exchanger of both (a), (b) diffluent and (c), (d) confluent at $V=100 \mathrm{~L} / \mathrm{h}$

Furthermore, the flow distribution in the Y-type heat exchanger is more even than that in the H-type. To quantify the impact of the maldistribution, a relative channel flowrate deviation $D_{c h}$ by numerical simulation was proposed to make a comparison of flow distributions ${ }^{[36]}, D_{c h}$ was defined as

$$
D_{c h}=\left(\frac{f_{c h}}{f_{a v}}-1\right) \times 100 \%
$$

Where $f_{c h}$ is the mass flowrate in a channel expressed in $\mathrm{kg} \mathrm{s}^{-1}$, and $f_{a v}$ is the calculated average flowrate in each channel expressed in $\mathrm{kg} \mathrm{s}^{-1}$. If the flow is completely uniform, then $D_{c h}=0$. A higher value of $D_{c h}$ represents a larger deviation compared to the uniform flow. Fig.6 presents the simulation results of $D_{c h}$ in different channels under different values of $\operatorname{Re}_{d}(648,3892,6488)$. It shows that the Y-type heat exchanger has a relative small deviation in the mass flowrate. With the increasing of $R e_{d}$, the fluid tends to be unstable, and the average deviation of flow distribution increases in each channel of the Y-type heat exchanger. The maximum deviation corresponding to each $\operatorname{Re}_{d}(648,3892,6488)$ is $3.76 \%, 10.27 \%$ and $12.31 \%$, 
respectively. Thus, under a low $R e_{d}$, a robust flow can be acquired in the Y-type channels.

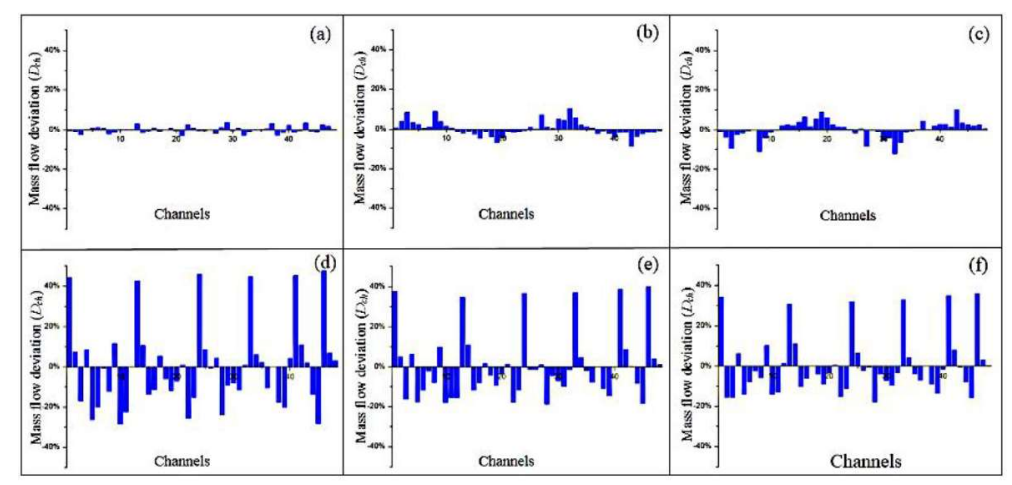

Fig.6 The mass flowrate deviation of each terminal channels of Y-type (a), (b), (c) and H-type (d), (e),

(f) under the different $R e_{d}$ of (a), (d) 648; (b), (e) 3892 and (c), (f) 6488

Compared with Y-type heat exchanger, the H-type heat exchanger also presents a much higher deviation of flow distribution, as shown in Fig.6 (d), (e) and (f). The current design organized H-type fractals into a framework of disk shape to make it compatible with the common chemical equipment. Beside the above discussion about the generation of the flow maldistribution, there is another reason for the significant uneven flow in the H-type channels. Under the low volumetric flow rate, most of the fluid would go through the channels with shorter length since the shorter flow length means less resistance. However, the flow resistance increases with the increasing of flow rate. When the flow rate rises to a certain degree, if the fluid still flows through the shorter length channel, it will cause a "flow jam" in the channel. To avoid flow jam and achieve a balance at steady state between momentum and energy dissipation in the flow, part of the fluid would pass through a longer length channel, leading to a decrease of $D_{c h}$ in each channel. The maximum deviation of the flow distribution corresponding to each $\operatorname{Re}_{d}(648,3892$, and 6488$)$ was $47.51 \%, 40.00 \%$, and $35.92 \%$, 
respectively. In addition, the maximum velocity in the H-type channels occurred at the center of the channel under confluent condition due to the inertia force and the large angle of circumfluence like the diffluent situation, thereby leading to an uneven flow in the channel. Meanwhile, the Y-type channels showed a well-mixed feature with a relatively uniform velocity profile [see Fig 5 (c, d)].

(a)

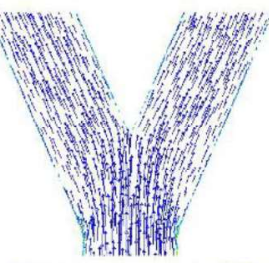

(b)

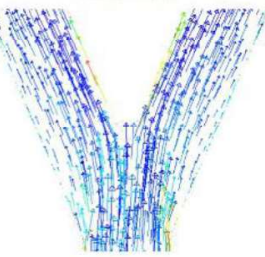

(c)

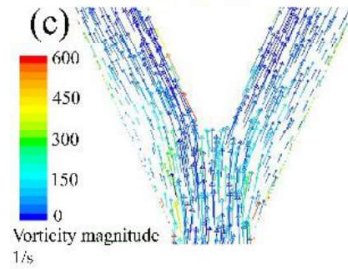

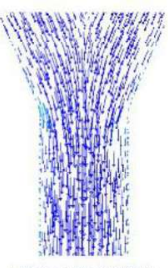
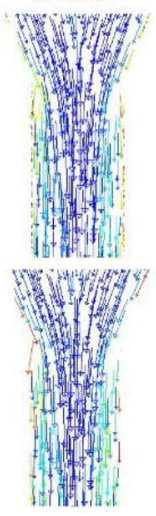
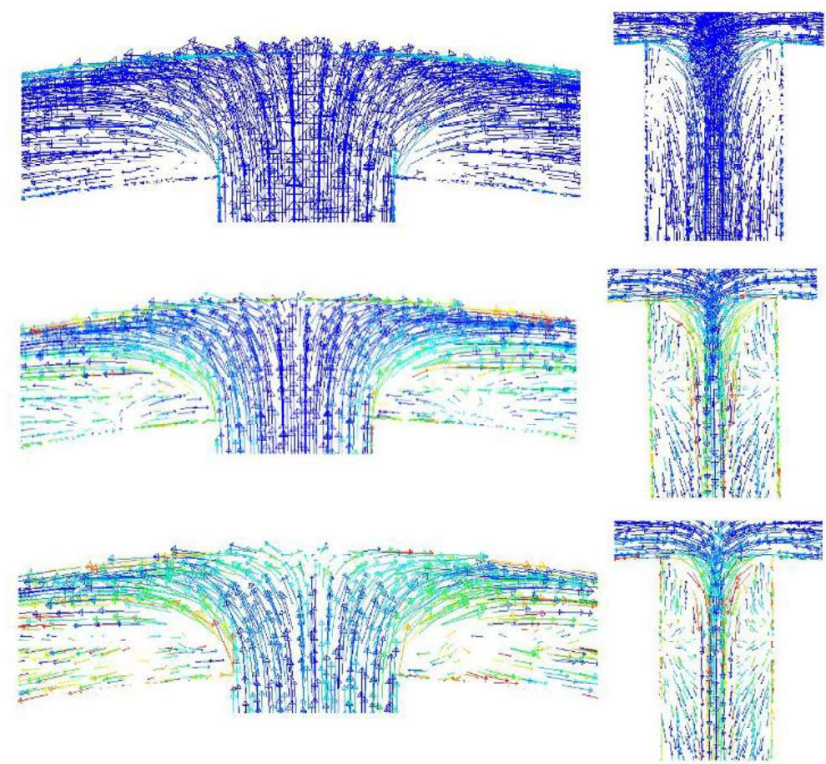

Fig.7 Comparison of the flow characterization and vorticity magnitude of $\mathrm{Y}$ and H-type heat exchanger in both diffluent and confluent point at different $R e_{d}$ : (a) 648, (b) 3892 and (c) 6488

Fig. 7 shows the simulated velocity vectors and vorticity magnitude of the first level bifurcation point in H-type and Y-type heat exchangers. These graphs illustrate the details of fluid mixing and separation process. As the fluid flowed through the bifurcation of the H-type heat exchanger, a secondary flow associated with vortexes was observed, while it was not found in the case of Y-type heat exchanger. In the case of H-type, there is no obvious vortex in the bifurcation channel at low flow rate. However, with the increasing flow rate, the viscous force drags the velocity of the fluids from the maximum at the center of the undisturbed mainstream to the zero at 
the channel wall. Compared with the mainstream, the fluid in the boundary layer has a strong deceleration effect. Therefore, the flow momentum of the fluid near the wall is too small to move forward when the pressure rises. Then, the mainstream flow slows down, unable to pull the fluid on the boundary layer to overcome the pressure rise and friction. Therefore, the fluid begins to leave the wall at a small angle, and a reverse flow occurs due to the adverse pressure gradient ${ }^{[17]}$. Moreover, a comparison of the vorticity magnitude with different $R e_{d}$ is also given in Fig.7 (a), (b), (c). With increasing $R e_{d}$, the vorticity magnitude in the H-type heat exchanger is increased in both diffluent and confluent conditions, owing to the higher adverse pressure gradient. In the case of the Y-type, the acute angle at the bifurcation tolerates the occurrence of secondary flow and vortexes; hence, a relatively more uniform distribution of velocity in the channel is presented, as shown in Fig. 5.

\subsection{Pressure drop}

Fig. 8 shows the pressure drop across the tube side as a function of the inlet volumetric flow rate, which was obtained from measurements and numerical predictions. In general, $\Delta P$ increases with the increasing volumetric flow rate. Among the three heat exchangers, the pressure drop of the traditional spiral-tube heat exchanger is much higher than those of the two novel fractal-tree-like heat exchangers. As discussed in the supporting information, the flow resistance in the fractal-tree-like network is lower than that in parallel channels and spiral-tubes. The Reynolds number in the spiral tube is between 2000 and 4000 once the volumetric flow rate rises from $30 \mathrm{~L} / \mathrm{h}$ to $60 \mathrm{~L} / \mathrm{h}$ in the transition region of laminar and turbulence flow. From the 
figure. 8 , it can be seen that a flow regime shifting can be identified for spiral-tube at $V=50 \mathrm{~L} / \mathrm{h}$. What's more, the quadratic shape of the curves for $\mathrm{Y}-$ and H-type exchangers is noticeable in the range of investigated flow rates, which is similar to the Dancy-forchheimer law used in porous media ${ }^{[37]}$.

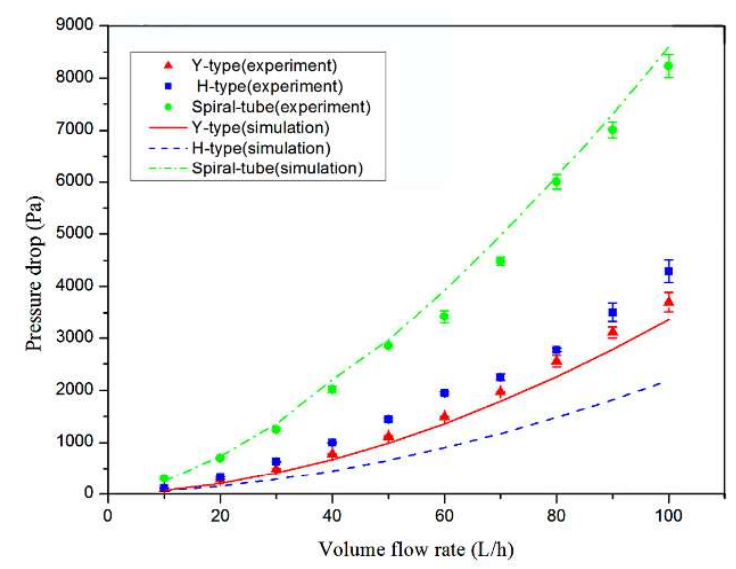

Fig.8 Comparison of pressure drop vs. volume flow rate for the three different types of heat exchangers in experiments and simulations

For the Y-type fractal and spiral-tube heat exchangers, a reasonably good agreement between the numerical prediction and mean values of experimental measurement was obtained. Generally, a slight deviation is inevitable. The maximum discrepancy is $13.4 \%$ and the mean error is approximately $5 \%$. In contrast, a large deviation between the numerical prediction and mean experimental measurements was observed in the H-type heat exchanger, as can be seen in Fig. 8. The simulation results of H-type heat exchanger show that the pressure drop value of the H-type heat exchanger is low, which is more suitable for the design of heat exchanger from the perspective of flow field distribution. Theoretically, the area to volume ratio of H-type exchanger is smaller than the Y-type, which means the structure of H-type is much 
more compact. What's more, the flow in the H-type channel is natural and spontaneous due to the balance of resistant and momentum. So the fluids don't have to flow across all the channels and suffer the additional pressure drop just like the Y-type channel. However, the experimental results show that the pressure drop of H-type channel is higher than Y-type, which is not compatible with the numerical results.

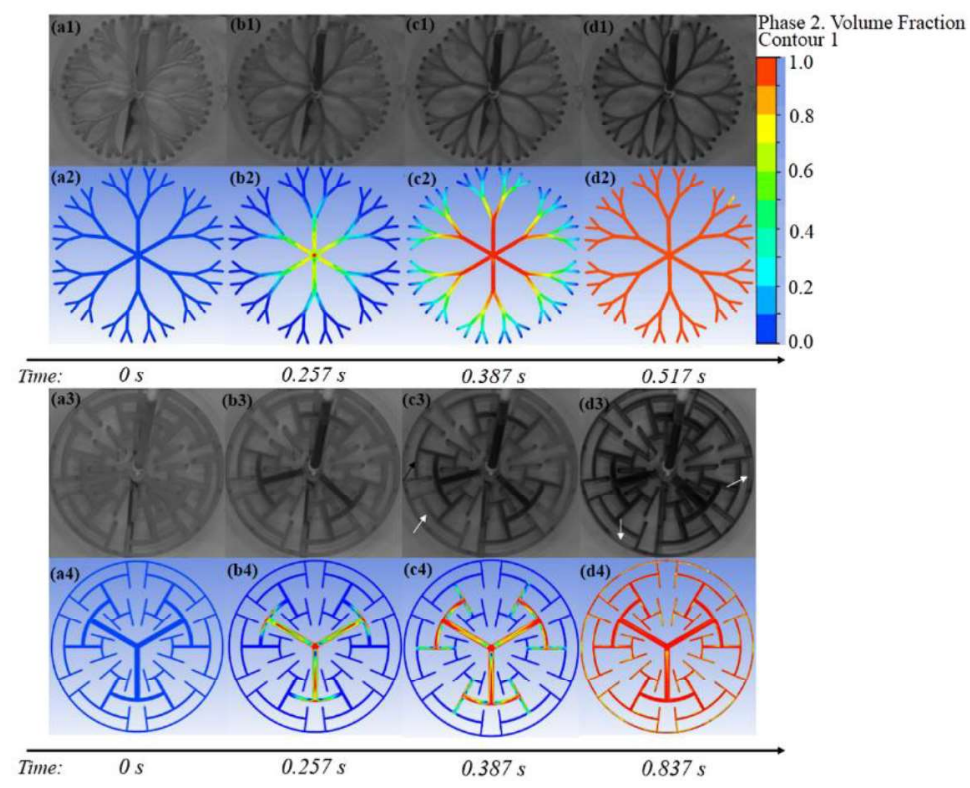

Fig.9 Comparison of experimental and simulation results of the flow development in Y-type and H-type heat exchangers at $V=100 \mathrm{~L} / \mathrm{h}$

To analyze the difference between numerical simulation and experiment, a visualization experiment was involved. The evolution of flow distribution in the H-type and Y-type heat exchangers was recorded using a high-speed monochrome camera (Phantom, PCC, Miro310) at 300 frames per second. Fig. 9 illustrates the distribution of the liquid dye and numerical simulation results from the main stream 
towards the bifurcation branches of the fractal-tree-like heat exchanger. Since the camera is monochromatic, the darker shade in the tube represents the liquid dye, which enters the main stream as shown in Fig. 9 (a1) and (a3). It then enters the first level of the fractal-tree-like channels, as shown in Fig. 9 (b1) and (b3). Next, the liquid further enters the subordinate branches; Uneven distributions of the liquid in the H-type heat exchanger are observed in Fig. 9 (c3). In the figure, within the same level of the tubes, the black arrow represents the tube filled with liquid dye, whereas the white arrow represents an empty tube. A similar uneven flow of the liquid is observed in the final level of the tubes [see Fig. 9 (d3)] of the H-type channels. However, the uneven flowing phenomenon is not observed in the Y-type heat exchanger according to Fig. 9 (a1, b1, c1, and d1). The development of the liquid dye through successive branches is always uniformly distributed. Finally, all the tubes are filled with liquid, as shown in Fig. 9 (d1). The experimental outcomes were verified with numerical results, as shown in Fig. 9 (a2, b2, c2, and d2).

In the H-type channels, the structure is more complicate and has a smaller channel diameter than Y-type channel. The H-type channel is more complicated than the Y-type channel and its channel diameter is smaller. Thus, the hierarchical structure of H-type exchanger makes it more difficult to remove the supporting materials from the 3D framework of H-type exchanger completely. Comparatively, it is easier for a 3D printer to reproduce an identical Y-type geometry as in the numerical case than H-type. Besides, the inner wall roughness of some channels in the H-type exchanger is rather higher than that in Y-type exchanger, increasing the flow resistance in certain 
channels, which leads to flow blind spots. Besides, any deviation in the positioning of the heat exchanger can cause additional resistance of the flow due to the gravity, which will deteriorate the robustness of the flow in the H-type channels and generating flow blind spots. It should be noted that, the structure of H-type may lack "robustness" with respect to slight imperfections, or to perturbations of the working conditions (clogging etc.) ${ }^{[38]}$. And even small irregularities in few channels of H-type exchanger would influence the flow in the high-level channel as well as the whole structure. The uneven flow of the liquid in the H-type heat exchanger leads to a major flow complexity (vortex, chaotic flow etc.) than initially computed numerically and introduces an additional pressure drop in the system. Partially, the deviation may be attributed to the laminar model applied in numerical simulation, which cannot fully capture the local vortex and turbulence observed in the H-type structure. Further studied will be conducted in our next work. This explains the formation of the deviation between the numerical prediction and measurement in Fig. 8.

\subsection{Dimensionless representation}

Some studies have reported that the fractal-tree-like structure reduced the transport distance and times, as well as the energy dissipation ${ }^{[12,39]}$. This is attributed to the compact design, which may affect the friction performance of the flow. The measured experimental value of the friction factor $f$ is a useful indicator in the comparison of different structures, and is defined as

$$
f=\frac{\Delta P}{l} \frac{d_{c}}{2 \rho u_{c}^{2}}
$$

Where $d_{c}$ is the characteristic length of the channel, $l$ is the length of the channel, 
and $u_{c}$ is the characteristic velocity of the channels. In this case, $d_{c}$ is defined as the inlet diameter, and $l$ is the streamwise channel length ${ }^{[40]}$. The value of $l$ for the $\mathrm{Y}, \mathrm{H}$, and spiral-type structures is $296.2 \mathrm{~mm}, 318.0 \mathrm{~mm}$, and $2894 \mathrm{~mm}$, respectively.

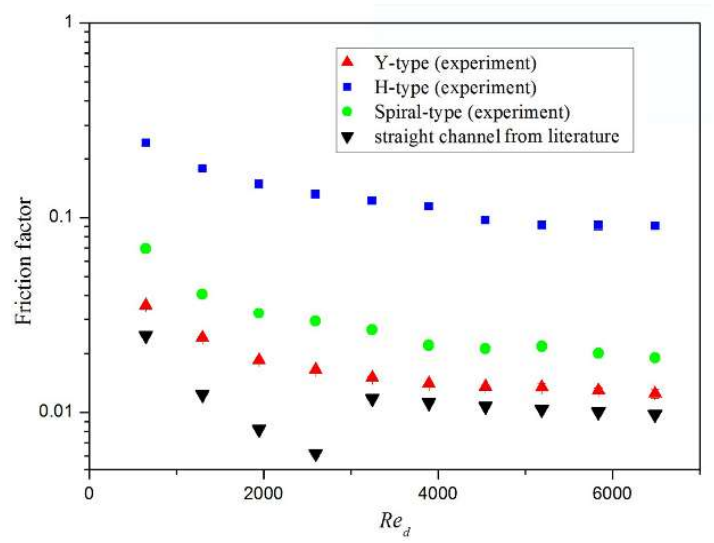

Fig.10 Comparison of the friction factors for the fluid in the channels with different structures

A comparison of the experimental friction factors among different heat exchanger structures is given in Fig. 10. A straight channel is also included and the value is calculated based on literature ${ }^{[41]}$. Notably, the friction factor of the H-type structure is the highest, which can be attributed to the vortexes and secondary flow in the H-type channels. The vortex increases local resistance, thus increasing the friction factor. Similar values and the tendency of the friction factor were also found in the T_network (in the range of $R e_{d} 648-1000$ ) and $\mathrm{x} \_$network (in the range of $R_{d} 1200$ - 5000) channels by Huchet et al. ${ }^{[42]}$, which has the similar structure to our H-type channels. Nevertheless, due to the compact structure of H-type channel, the fluids flow through a shorter path and suffer less resistant. Thus, the pressure drop of H-type channel is still lower than the traditional spiral-tube. In the case of the spiral-tube, the centrifugal force leads to the fluid moving toward one side (which can be seen in 
Figure. S2), hence, it intensifies the shear stress between the fluid and tube inner wall, leading to the second highest value of friction factor. The friction factor of the Y-type is lower than that of both the H-type and spiral tubes, because the fluid flows more uniformly across the channel than in the other two. However, the value is still higher than that in a straight channel. As mentioned, the flow in the branching channel is difficult to develop fully owing to the short developing path length. Generally, the friction factor of unsteady flow is higher than that of fully developed flow in the long straight channel. Nevertheless, with the increasing Reynolds number $\left(\operatorname{Re}_{d}>2500\right)$, the flow in straight channels turns to the turbulent state and its friction factor increases correspondingly, meanwhile the flow in the channel of the fractal-tree-like heat exchanger is still in the laminar state, as discussed above $\left(\operatorname{Re}_{l}<2000\right)$. The disparity of friction factors among fractal-tree-like heat exchangers is reduced since the friction factor keeps decreasing.

However, among the Y-type, H-type, and spiral-tube heat exchangers, the difference in the friction factor is substantial in the range of low Reynolds number. This can be partly attributed to the large difference in temperature between the inlet and outlet of the heat exchanger. With the increase of Reynolds number, the fluid velocity increases; thus, the residence time decreases and the temperature difference of inlet and outlet becomes smaller. Consequently, the influence of temperature on the friction factors declines. Similar results were also reported by Toh et al. ${ }^{[43]}$ and Liu et al. ${ }^{[44]}$. In their studies, at a lower Reynolds number, the fluid temperature is increased more rapidly, which leads to a decrease in viscosity, and hence reduces the frictional 
loss.

\subsection{Heat transfer performance}

According to the law of energy conservation, the rate of heat flow $\mathrm{Q}$ can be calculated as following

$$
Q=m c_{p}\left(T_{0}-T_{1}\right)
$$

Where $m$ represents the mass flow rate, $T_{0}$ and $T_{1}$ represent the inlet and outlet temperatures of hot water, respectively. The total heat flux can be expressed as

$$
q=\frac{Q}{A_{m}}=\frac{m c_{p}\left(T_{0}-T_{1}\right)}{A_{m}}
$$

A comparison of the total heat flux between the two fractal-tree-like heat exchangers (H-type and Y-type) and the traditional spiral-tube heat exchanger is shown in Fig. 11. In general, the total heat flux increases with an increase in the Reynolds number monotonically, and this trend is more pronounced at lower Reynolds numbers (i.e., $\mathrm{Re}<3000$ ). A reasonably good agreement was obtained between the numerical value and experimental data, with a mean value of $6 \%$ and a maximum value of $8.6 \%$. The small discrepancy can be attributed to the ideal physical conditions assumed in the simulations, such as adiabatic system and negligible heat radiation. It is noteworthy that the discrepancy increases with increasing Reynolds number, particularly in the H-type heat exchanger. Based on the experimental and numerical results, it is clear that the H-type heat exchanger provides the highest total heat flux, and the Y-type and spiral-tube heat exchangers offer similar values for the total heat flux. 


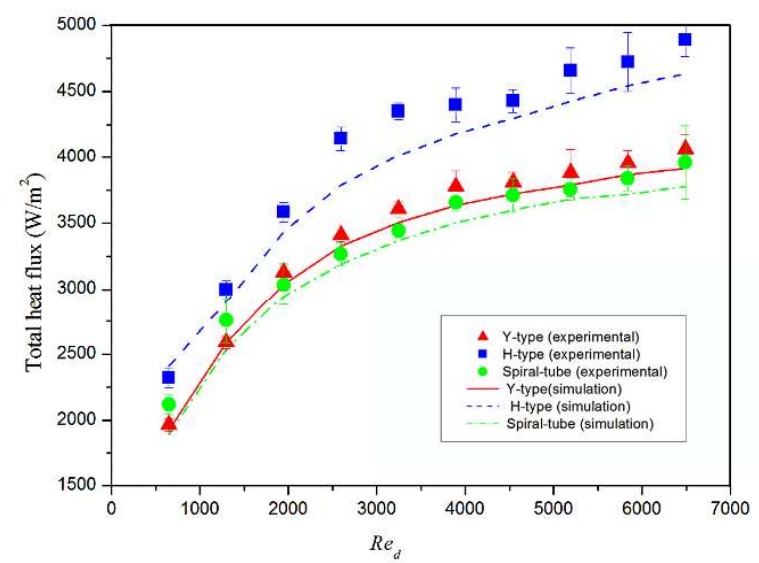

Fig.11 Total heat flux as a function of $R e_{d}$

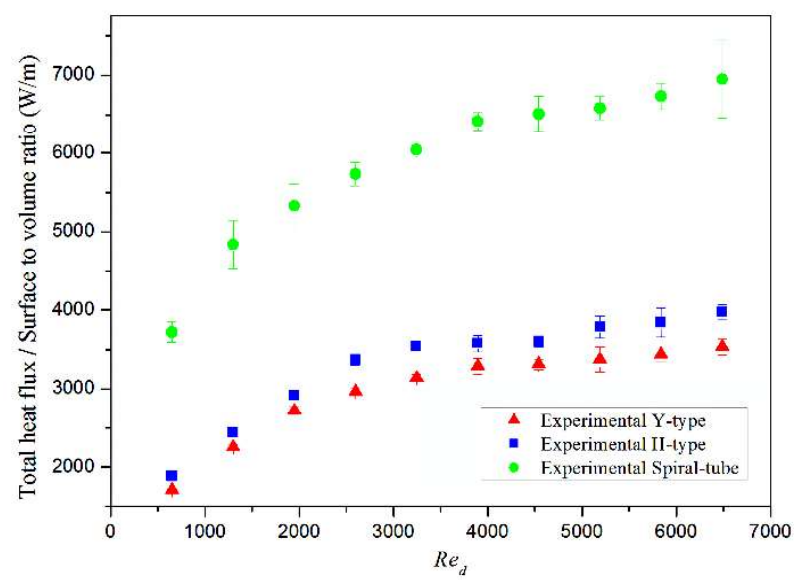

Fig.12 Experimental total flux scaled by surface to volume ratio

As discussed before, the area to volume ratio among three types of heat exchanger are quite different. To avoid the influence of area to volume ratio, the experimental data of total heat flux is scaled by the area to volume ratio and the results are shown in Fig. 12. Contrastively, the total heat flux of Spiral-tube heat exchanger is the highest excluding the effect of area to volume ratio. It means that the compact fractal-tree-like structure with high area to volume ratio is more beneficial to heat transfer, compared with traditional Spiral-tube heat exchanger. In other words, the area to volume ratio plays an essential role in the process of heat transfer. 
It also can be seen that the value of H-type heat exchanger is still higher than Y-tube one, so the mixing and vortexes of flow are considered another important factors affecting heat transfer capability. The velocity profile and the effect of structure in the generation of vortex is shown in Fig. 7 ( $a, b$ and $c$ ). In the H-type channels, secondary flow and vortexes exist in both splitting and merging bifurcations. The existence of secondary flow and vortexes can intensify the heat transfer between low temperatures close to the tube wall and high temperatures at the tube center, which can also enhance the laminar mixing. It thus explains why the H-type exchanger has a better thermal performance than Y-type exchangers. Nevertheless, it should be pointed out that the vortexes may cause additional pressure drops in the network. However, the overall pressure drop of H-type heat exchanger is still less than that of the Spiral-tube heat exchanger, as shown in Fig. 8.

\subsection{Coefficient of performance}

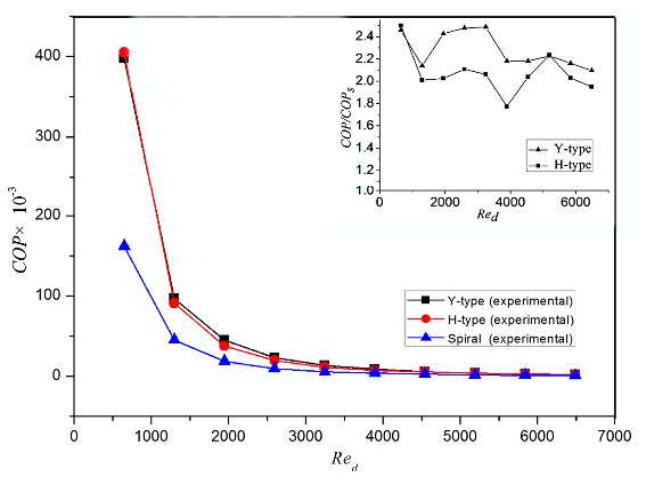

Fig.13 Comparison of the coefficient of performance (COP) among three types of heat exchanger

As discussed above, experimentally, the H-type exchanger has a greater thermal performance, whereas the Y-type exchanger has a better hydrodynamic performance since it has a smaller pressure drop in the system. Hence, a synthesized index is 
introduced to evaluate the overall coefficient of performance $(C O P)$ for the heat exchangers. The experimental $C O P$ can be defined as the ratio of thermal performance to pumping power ${ }^{[12]}$,

$$
C O P=\frac{Q}{\Delta P V}
$$

Where $V$ is the volumetric flow rate. The comparison of experimental $C O P$ of each heat exchanger is shown in Fig. 13. It indicates that the COP decreases with the increase in Reynolds number. It also illustrates that the flow resistance has significant influence on the comprehensive performance. With the increasing Reynolds number $\left(\operatorname{Re}_{d}>3500\right)$, it is difficult to distinguish the three types of heat exchangers. To compare the performance between H-type and Y-type heat exchangers further, the values of experimental $C O P / C O P_{s}$ are plotted as a function of the Reynolds number, and attached as an inset in the Fig. 13, where $C O P_{s}$ is the $C O P$ of the spiral-tube heat exchanger. As shown in Fig. 13, the value of $C O P / C O P_{s}$ is always greater than 1.0, which means that both fractal-tree-like heat exchangers are better than the spiral-tube exchanger in the experimental conditions, and the Y-type exchanger outperforms the H-type heat exchanger, in general. As discussed earlier, the H-type heat exchanger provides higher total heat flux; however, its high pressure drop outweighs this advantage, and results in a lower $C O P$ than that of the Y-type.

\section{Conclusion}

Two novel fractal-tree-like and one traditional spiral-tube heat exchangers were designed and manufactured by $3 \mathrm{D}$ printing that enables the precise and fast fabrication of complicated three-dimensional structures such as fractal-tree-like 
devices.

The model employed in the present work was verified using an SLA Y-type fractal-tree-like heat exchanger. The numerical method was proven accurate in Y-type channels. Meanwhile, it can be concluded that the application of 3D printing technology is an effective method for manufacturing a heat exchanger as it reduces the cost and time. This type of printing provides an efficient, accurate, and precise approach toward the fabrication of a complex structure, and will bring great potential applications in the chemical industry. Combined with CFD simulations, 3D printing enables quick and efficient research into a structure-activity relationship.

Although vortexes were observed in H-type channels, which are associated with higher friction factor, the heat exchanger based on the fractal network can reduce the systematic pressure drop and enhance the heat transfer performance compared with a conventional spiral tube heat exchanger, based on the experimental and numerical results. In addition, a heat exchanger based on a fractal network has a higher $C O P$ than the conventional heat exchanger under the present experiment conditions.

\section{Acknowledgements}

This work was supported by the Youth Innovation Promotion Association of Chinese Academy of Sciences and the Key Technical Personnel of Chinese Academy of Sciences, the Ministry of Science and Technology of China (grant 2016YFA0602603). The work was also supported by Frontier Scientific Research Project funded by Shell under contract No. PT19253.

\section{Notations}


$A_{m}=$ heat transfer area, $\mathrm{m}^{2}$

$C F D=$ Computational Fluid Dynamics

$C O P=$ coefficient of performance

$C_{p}=$ thermal capacity, $\mathrm{J} \mathrm{kg}^{-1} \mathrm{~K}^{-1}$

$D=$ deviation

$d=$ diameter, $\mathrm{m}$

$d_{i 1,2}=$ diameter of one branch in level $i, \mathrm{~m}$

$f=$ friction factor

$f_{c h}=$ mass flowrate in a channel, $\mathrm{kg} \mathrm{s}^{-1}$

$f_{a v}=$ average mass flowrate, $\mathrm{kg} \mathrm{s}^{-1}$

$H=$ H-type heat exchanger

$I E A=$ International Energy Agency

$k=$ number of branches of a series

$l=$ length, $\mathrm{m}$

$m=$ mass flow rate, $\mathrm{kg} / \mathrm{s}$

$P M M A=$ polymethyl methacrylate

$Q=$ heat transfer quantity, $\mathrm{W}$

$q=$ total heat flux, $\mathrm{W} \mathrm{m}^{-2}$

$R^{+}=$characteristic variable

$R e=$ Reynolds number

$R e_{d}=$ device Reynolds number

$R e_{l}=$ local Reynolds number 


$$
\begin{aligned}
& S=\text { cross-sectional area, } \mathrm{m}^{2} \\
& T=\text { temperature } \mathrm{K} \\
& U V=\text { ultraviolet } \\
& u=\text { velocity, } \mathrm{m} \mathrm{s}^{-1} \\
& V=\text { volume flow rate, } \mathrm{L} \mathrm{h}^{-1} \\
& v=\text { kinematic viscosity, } \mathrm{m}^{2} \mathrm{~s}^{-1} \\
& W=\text { total uncertainties in measurement } \\
& Y=\text { Y-type heat exchanger } \\
& \Delta P=\text { pressure drop, Pa }
\end{aligned}
$$

\section{Greek letters}

$\theta=$ included angle between two branches, ${ }^{\circ}$

$\mu=$ Dynamic viscosity, Pa.s

$\lambda=$ thermal conductivity, $\mathrm{W} \mathrm{K}^{-1} \mathrm{~m}^{-1}$

$\rho=$ density, $\mathrm{kg} \mathrm{m}^{-3}$

$\Delta=$ fractal dimension

\section{Subscripts and superscripts}

$$
\begin{aligned}
& d=\text { device } \\
& i=\text { branch level } \\
& l=\text { local } \\
& s=\text { spiral } \\
& 0=\text { state before heat transfer }
\end{aligned}
$$


$1=$ state after heat transfer

\section{References}

[1] International Energy Agency. Key world energy statistics 2015. Paris: IEA, 2015.

[2] International Energy Agency. $\mathrm{CO}_{2}$ emissions from fuel combustion (2015 Edition). Paris: IEA, 2015.

[3] Li K, Leigh W, Feron P, et al. Systematic study of aqueous monoethanolamine (MEA)-based $\mathrm{CO}_{2}$ capture process: techno-economic assessment of the MEA process and its improvements [J]. Applied Energy. 2016; 165:648-659.

[4] Al-Kalbani H, Xuan J, García S, et al. Comparative energetic assessment of methanol production from $\mathrm{CO}_{2}$ : chemical versus electrochemical process [J]. Applied Energy. 2016; 165:1-13.

[5] Bejan A, Errera MR. Deterministic tree networks for fluid flow: geometry for minimal flow resistance between a volume and one point [J]. Fractals. 1997; 5 (04):685-695.

[6] Kulkarni AA, Jha N, Singh A, et al. Fractal Impeller for Stirred Tank Reactors [J]. Industrial \& Engineering Chemistry Research. 2011; 50 (12): 7667-7676.

[7] Luo L, Min W, Fan Y, et al. Heuristic shape optimization of baffled fluid distributor for uniform flow distribution [J]. Chemical Engineering Science. 2015; 123: 542-556.

[8] Guo X, Fan Y, Luo L. Multi-channel heat exchanger-reactor using arborescent distributors: a characterization study of fluid distribution, heat exchange performance and exothermic reaction [J]. Energy. 2014; 69:728-741.

[9] Bejan A. The tree of convective heat streams: its thermal insulation function and the predicted 3/4-power relation between body heat loss and body size [J]. International Journal of Heat \& Mass Transfer. 2001; 44 (4):699-704. 
[10] Chen Y, Cheng P. Heat transfer and pressure drop in fractal-tree-like microchannel nets [J]. International Journal of Heat \& Mass Transfer. 2002; 45(13):2643-2648.

[11] Chen Y, Cheng P. An experimental investigation on the thermal efficiency of fractal tree-like microchannel nets [J]. International Communications in Heat \& Mass Transfer. 2005; 32(7):931-938.

[12] Chen Y, Zhang C, Shi M, et al. Thermal and hydrodynamic characteristics of constructal tree-shaped minichannel heat sink [J]. AIChE Journal. 2010; 56 (8):2018-2029.

[13] Yu XF, Zhang CP, Teng JT, et al. A study on the hydraulic and thermal characteristics in fractal tree-like microchannels by numerical and experimental methods [J]. International Journal of Heat \& Mass Transfer. 2012; 55(s 25-26):7499-7507.

[14] Guo X, Fan Y, Luo L. Mixing performance assessment of a multi-channel mini heat exchanger reactor with arborescent distributor and collector [J]. Chemical Engineering Journal. 2013;227 (7):116-127.

[15] Coppens M O. A nature-inspired approach to reactor and catalysis engineering[J]. Current Opinion in Chemical Engineering, 2012, 1(3):281-289.

[16] Chu JC, Teng JT, Xu TT, et al. Characterization of frictional pressure drop of liquid flow through curved rectangular microchannels [J]. Experimental Thermal \& Fluid Science. 2012;38 (4):171-183.

[17] Zhang CP, Lian YF, Hsu CH, et al. Investigations of thermal and flow behavior of bifurcations and bends in fractal-like microchannel networks: Secondary flow and recirculation flow [J]. International Journal of Heat and Mass Transfer. 2015; 85:723-731.

[18] Alharbi AY, Pence DV, Cullion RN. Thermal characteristics of microscale fractal-like branching channels [J]. Journal of Heat Transfer. 2004; 126 (5):744-752.

[19] Bhargava KC, Bryant T, Noah M. Discrete elements for 3D microfluidics [J]. Proceedings of the 
National Academy of Sciences of the United States of America. 2014; 111 (42):15013-8.

[20] Huchet F, Comiti J, Tihon J, et al. Electrodiffusion diagnostics of the flow and mass transfer inside a network of crossing minichannels [J]. Journal of Applied Electrochemistry. 2007; 37 (1):49-55.

[21] Ben-Ner A, Siemsen E. Decentralization and Localization of Production: The Organizational and Economic Consequences of Additive Manufacturing (3D Printing)[J]. California Management Review, 2017:000812561769528

[22] Monzel WJ, Hoff BW, Maestas SS, et al. Dielectric breakdown of additively manufactured polymeric materials [J]. IEEE Transactions on Dielectrics \& Electrical Insulation. 2015; 22 (6):3543-3549.

[23] Murray CD. The physiological principle of minimum work I: the vascular system and the cost of blood volume [J]. Proceedings of the National Academy of Sciences of the United States of America. 1926; $12(3): 207-214$

[24] BenoitB. The fractal geometry of nature [M]. W.H. Freeman, 1983.

[25]Xu S, Wang W, Fang K, et al. Heat transfer performance of a fractal silicon microchannel heat sink subjected to pulsation flow [J]. International Journal of Heat \& Mass Transfer. 2015; 81 (81): 33-40.

[26] Senn SM, Poulikakos D. Laminar mixing, heat transfer and pressure drop in tree-like microchannel nets and their application for thermal management in polymer electrolyte fuel cells [J]. Journal of Power Sources. 2004; 130 (1-2):178-191.

[27] Kumar R, Sahoo N, Kulkarni V. Conduction based calibration of handmade platinum thin film heat transfer gauges for transient measurements [J]. International Journal of Heat \& Mass Transfer. 2012; 55 (9-10):2707-2713.

[28] Sakai N, Fujii K, Konakahara T. Dynamic calibration of a coaxial thermocouples for short 
duration transient measurements [J]. Journal of Heat Transfer. 2013; 135 (12):216-249.

[29] Handbook of Chemistry and Physics (82nd edition). Boca Raton, FL: CRC Press, 2002.

[30] Squires T. Microfluidics: fluid physics at the nanoliter scale [J]. Reviews of Modern Physics. 2005; 77 (3):977-1026.

[31] Moffat RJ. Describing the uncertainties in experimental results [J]. Experimental Thermal \& Fluid Science. 1988; 1 (1):3-17.

[32] Herwig H, Gloss D, Wenterodt T. A new approach to understanding and modelling the influence of wall roughness on friction factors for pipe and channel flows[J]. Journal of Fluid Mechanics, 2008, 613(613):35-53.

[33] Chen Y, Zhang C, Shi M, et al. Role of surface roughness characterized by fractal geometry on laminar flow in microchannels.[J]. Physical Review E Statistical Nonlinear \& Soft Matter Physics, 2009, 80(2):026301.

[34] Gamrat G, Favremarinet M, Person S L, et al. An experimental study and modelling of roughness effects on laminar flow in microchannels[J]. Journal of Fluid Mechanics, 2008, 594(594):399-423.

[35] Kandlikar S G, Schmitt D, Carrano A L, et al. Characterization of surface roughness effects on pressure drop in single-phase flow in minichannels[J]. Physics of Fluids, 2005, 17(10):133-98.

[36] Fan Y, Boichot R, Goldin T, et al. Flow distribution property of the constructal distributor and heat transfer intensification in a mini heat exchanger [J]. AIChE Journal. 2008; 54 (11):2796-2808.

[37] Soulaine C, Quintard M. On the use of a Darcy-Forchheimer like model for a macro-scale description of turbulence in porous media and its application to structured packings[J]. International Journal of Heat \& Mass Transfer, 2014, 74(7):88-100.

[38] Luo L, Tondeur D, Gall H L, et al. Constructal approach and multi-scale components[J]. Applied 
Thermal Engineering, 2007, 27(10):1708-1714.

[39] West G B, Brown J H, Enquist B J. The fourth dimension of life: fractal geometry and allometric scaling of organisms [J]. Science, 1999, 284 (5420):1677.

[40] Li P, Xie Y, Zhang D. Laminar flow and forced convective heat transfer of shear-thinning power-law fluids in dimpled and protruded microchannels[J]. International Journal of Heat \& Mass Transfer, 2016, 99:372-382.

[41] McCabe, WarrenL. Unit operations of chemical engineering. 5th edition [M]. McGraw-Hill Book, 1993: p.101

[42] Huchet F, Comiti J, Legentilhomme P, et al. Mixing characterization and energetic dissipation in different networks of minichannels [J]. Chemical Engineering Research \& Design. 2008; 86 (10A):1135-1142.

[43] Toh K C, Chen X Y, Chai J C. Numerical computation of fluid flow and heat transfer in microchannels [J]. International Journal of Heat \& Mass Transfer, 2002, 45 (26):5133-5141.

[44] Liu C, Teng J T, Chu J C, et al. Experimental investigations on liquid flow and heat transfer in rectangular microchannel with longitudinal vortex generators [J]. International Journal of Heat \& Mass Transfer, 2011, 54 (13-14):3069-3080. 